a subject which cannot be cleared up without much future investigation, I may yet say that I incline to believe the origin of Addison's disease in many of the unexplained cases to be due to traumatic causes, although probably favoured in its development by certain constitutional proclivities.

Very few words need be said here with respect to the diagnosis and prognosis of Addison's disease. The diagnosis is founded upon the constitutional symptoms, aided, in a large majority of cases, by the presence of more or less of the peculiar change of colour in the skin. It is not always unattended with difficulty, but to those who have any practical acquaintance with the disease it is not, I think, more doubtful than the diagnosis of many other chronic diseases. The prognosis is, of course, invariably grave as regards the ultimate result, though it is impossible to say to what extent life may be prolonged under favourable circumstances. Rest and scrupulous avoidance of bodily and mental excitement, or any other causes of nervous exhaustion, form the essential parts of the therapeutic management of all such cases; whilst the diet and medical treatment must be carefully adapted to the inevitably varying phases of the disease.

It only remains for me to sum up, in conclusion, the objects I have had in view in the course of lectures which I have had the honour to deliver before you.

In the first, I believe, I delineated faithfully all the principal clinical symptoms of Addison's disease and the remarkable varieties in their course, together with the true characters of the pathological lesions in and around the suprarenal capsules, which have been found to coexist with them.

In the second lecture $I$ endeavoured to show clearly, on the one hand, the concurrent testimony of facts in proof of the real connexion subsisting between these clinical symptoms and the one specific lesion in the supra-renal capsules, and, on the other hand, the baseless nature of the misconceptions which have prevented the general recognition of its reality.

In to-day's lecture I have trodden on more difficult ground. No one can feel more strongly than myself that the opinions I have been led to form, with respect to the obscure pathological and etiological processes in Addison's disease, rest as yet upon an inadequate basis. I can only express the hope that, however problematical their correctness may appear to many, the suggestions I have ventured to make regarding the probable mode of production of the symptoms, and the probable means of origin of the supra-renal disease itself, may lead to a thorough investigation, in future cases, of all the facts bearing upon these questions; and thus to the acquisition of knowledge which may justify positive conclusions, in place of the uncertain inferences which, on many points, are all I have been able to draw from $m y$ own necessarily limited personal experience and the insufficient materials at uny cornmand.

STAB THROUGH THE EAR; WOUND OF MIDDLE MENINGEAL ARTERY; TRAU. MATIC ANEURISM; LIGATURE OF LEFT COMMON CAROTID ARTERY; DEATH.

Bx SAMPSON GAMGEE, F.R.S.F., SURGEON TO THE QUEEN'S HOSPTTAL, BIRMINGHAM,

WILIIAM LINES, police-constable in the Birmingham force, was admitted into the Queen's Hospital, under my care, on March 7 th, faint and pulseless. Bright-red blood was flowing profusely from a clean incised wound through the tragus and along the anterior wall of the external auditory meatus and the tympanum of the left side. After clearing out the clots, a graduated compress was applied, and the patient put to bed with an ice-bag over the left side of the head. Slight hæmorrhage, recurring during the night, was stopped by the nurse pressing on the pad. The next morning reaction had fully set in, and the patient suffered very little pain. Pulse 84 ; temperature $100^{\circ} \mathrm{F}$.

March 10th.-Temperature $994^{\circ}$; pulse 76.

changed; no hrmorrhage. Ice to be continued.

Ear plug

13th.-No more hæmorrhage since the first night. The patient is so much better that the magisterial depositions are taken at the bedside.

16th.-Palse 76. Asked for chicken for dinner.

17th. - A swelling below the ear, which has gradually developed, pulsates, and is the seat of bruit. At 10.30 P.M. smart hæmorrhage from the ear, stopped by replugging Pressure by the nurse on the plug was sufficient to check subsequent slight bleeding. The application of ice continued without intermission.

19th.-The pulsating swelling about the ear increasing digital compression of the left common carotid resolved upon, and its applieation was undertaken and carried out by clinical stidents, Messrs. Butt, Cresswell, Dennis, Dumbleton, F、llows, Gascoigne, Godson, Hall, Johnston, Palmer, Percival, Rhodes, and Tomkinson. The pulsation and bruit very perceptibly lessened, with consolidation of the tumour, but as the improvement was not persistent as digital pressure became unbearable, and, after slight bleedings, considerable hæmorrhage occurred, at 4 P.M. on the 23rd of March I ligatured the common carotid as the only means of prolonging life. After ligature and the emptying of the aneurism, the hæmorrhage definitely ceased, and the patient soon rallied to perfect consciousness, and asked for his wife and child. Four hours later he was delirious. Eight hours and a half after the operation the right side became paralysed, and death occurred at noon on March 24th.*

The post-mortem examination showed the catgut ligature firmly embracing the left common carotid just below the omo-hyoid; on opening the artery, the inner coat was cleanly divided, and a firm adherent clot plugged the proximal extremity; the distal side of the vessel was quite empty. The wound in the ear perforated the membrana tympani, and an irregular cavity, between and about the pterygoid muscles, was filled with semi-purulent decomposing clot. On removing the calvaria, the branches of the left middle meningeal vessels were quite empty, showing a marked contrast to their distended condition on the opposite side; the dura mater and brain healthy. The internal carotid in the skull and petrous bone was laid bare, and found free from injury. The external carotid and its branches having been previously dissected and found sound, the middle meningeal artery was traced from the internal maxillary through the foramen spinosum, and outwards along the base of the skull for about a quarter of an inch, where it was found to be torn in consequence of the splintering of the thin lamina of bone at the junction of the squamous and petrous portion of the temporal. From the under surface of this part of the skull, an irregular sloughing canal passed down to the false aneurismal sac between the pterggoid muscles, along which the blood had passed from the injured middle meningeal artery, being prevented from separating the dura mater by the close adbesion of that membrane in the base of the skull. All the other branches of the external carotid were healthy and free from injury.

Commentary. -A stab into the ear followed by protuse hæmorihage would, under any circumstances, be attended with danger. Pressure, ice, and perfect rest, were the first means employed to arrest bleeding, and, for some days, with success. When the hæmorrhage recurred, and a pulsating tumour gradually developed in front and below the wounded ear, the question of further interference pressed for solution, and the attendant responsibility was increased by the reflection that, as the wound was stated to have been inflicted on the constable while he was in the discharge of duty, a charge of wilful murder was impending, and two lives might be involved in the issue. It was obviously impossible to say with precision what artery was wounded, and the position of the injury precluded resort to the practice of cutting down in search of the wounded vessel, and tying it in situ. The recurring hæmorrhage was slight in amount; pressure on the common carotid instantly checked $i t$, and at the same time arrested the pulsation and bruit in the aneurism. Experience is steadily accumulating in favour of the treatment of aneurism by digital compression-a plan which in itself is singularly harmless. Ligature of the common carotid is attended with its own risk. These considerations decided me in giving

* This abstract is from full clinical notes taken by our house-surgeon, Mr. F. G. Hamilton, whose ability and prudence were very notable throughout the case. For the notes of the autopsy I am indebted to my friend and colleague Mr. William Thomas. 
digital compression a thorough trial一a decision in which I was sustained by my colleagues in consultation. An appeal was no sooner made to our students for volunteers to practise digital compression than a number came forward, and it is difficult to do justice to the gentle, faithful, and skilful care with which these gentlemen, over a period of seventy-two hours, remained at their post. For a time their endeavours promised the best result: the swelling grew solid, the pulsation and blowing murmur very perceptibly decreased; but eventually bright scarlet blood flowed again, and to check it it was necessary to exercise increasing pressure, which the patient was less able to bear. Death from hæmorrhage now being imminent and certain, I resolved to tie the common carotid as the only means of prolonging life. Dr. Sawyer administered ether, and $\mathrm{Mr}$. West and $\mathrm{Mr}$. Wilders assisted $\mathrm{me}^{*}$ while $\mathrm{I}$ cut down upon the common carotid, and secured it with a catgut ligature just below the omo-hyoid. 'The man's thick and very muscular neck added to the difficulty of the operation in this deep position; but I deemed it safest to cut through healthy tissues, well below the infiltration in and below the parotid region. For the immediate object for which it was performed-the arrest of hæmorrhage which threatened instant death - the operation was perfectly successful, although the supervention of hemiplegia and pulmonary congestion proved fatal in twenty hours.

Birmingham.

\section{ON THE FORMATION OF STONE IN THE BLADDER AFTER INJURY TO THE SPINE.}

BY HENRY A. I,EDIARD, M.B.,

AYEDICAT OFFICER OF THE CLBVELAND-STRHET ASYLUM, IN THE CENTRAL LONDON SICK ASYLUM DISTRICT.

Chronic crstits is very commonly found in the paraplegic, and in such cases the urine will possibly be turbid, with an alkaline reaction, together with the presence of muco-pus and crystals of triple phosphate; yet I believe those cases far from ordinarily met with where such a state ultimately leads to the formation of a calculus in the bladder. The same condition of the urine may exist from any cause which delays the exit from the body of water excreted healthily by the kidneys. Or, again, the presence of a calculus in a normal bladder may set up a chronic cystitis which eventually coats the stone secondarily with phosphatic material; and the same may be said with regard to foreign bodies in the bladder, which are treated in a similar manner, the last specimen of the kind I saw being an extensive phosphatic deposit upon a hair-pin, removed from the bladder of a young female. In spinal injuries followed by paraplegia, the cystitis set up has been attributed to loss of nutrient power of the tissues, the paralysed state of the bladder rendering this viscus more prone to inflam. mation. It is difficult to account for the fact that chronic cystitis may exist in some instances for a long while, and no concrement form at any time; whereas, in a few cases a stone may form at an early date, but I suspect it is due to this, that in the former class of cases the bladder is frequently emptied, and that the latter class of cases are associated with deficiency in expulsive power of the bladder.

In paralysis of the bladder, where the sphincter retains power and the detrusor is chiefly affected, the conditions most favourable to the formation of stone are presented: the urine is allowed to accumulate within the viscus, the pain of distension is not experienced perhaps, and it is only when over-distension ensues that the urine begins to dribble; the long residence within the bladder induces concentration of the urine, and aggravates the deposit thrown down. Were this paralysis of the detrusor more commonly met with together with chronic disease of the bladder, I believe that cases such as the one I record would be of more frequent occurrence.

I am enabled, through the courtesy of Mr. Holden, to

* My colleague, Mr. Furneaux Jordan, who had previously given me th full benefit of his opinion, was unavoidably absent at the moment of the operation. give an account of the injury sustained by the patient and consequences that ensued.

W. $G-$, aged thirty-nine, was admitted into St. Bartholomew's Hospital on Jan. 23rd, 1873, with an injury to the spine. He had been moving some waggons, when one of them struck the corver of a goods shed, a great portion of which fell, striking him across the back and loins. There was tenderness on pressure and ecchymosis over the lower dorsal and upper lumbar vertebræ. No fracture or dislocation could be detected. The urine was obliged to be drawn off. The motion of both legs was impaired, especially that of the lef $t$. He complained of numbness in both legs. Bowels acted involuntarily. Subsequently, on Feb.2nd, the urine became thick and ammoniacal. On the 5th it contained blood. He had a rigcr, and the pulse and temperature rose. Pus and crystals of triple phosphates appeared in the urine. On the 13th there was more movenent in both legs. On March 18th he had passed for himself, as often as he had wanted it, a soft catheter, and the bladder had been occasionally washed out. He was able to sit up by the fire on March 27th. He could not pass urine, but motions were voided before he could ask for a utensil. On May 19th he left the hospital on crutches, and he told me that he relinquished these in three weeks in favour of a stick, which be used up to his admission into the Highgate Infirmary on Dec. 4th, 1874, when he made the following statement: that he had injured his spine two years since, that he had used a catheter daily for the last fifteen months, and that a swelling began to make its appearance between the legs ten days before admission.

Upon examination, there is an abnormal interval between the spinous processes of the last dorsal and first lumbar vertebræ, with considerable prominence of the latter; expression anxious, thirst present, and a large fluctuating tumour in the perineum, a mesial incision into which evacuated twelve ounces of grumous pus stinking horribly. The cavity of the abscess was then washed out, and lined with lint and carbolic oil; the scrotum suspended. In the evening the sheets were wet under him, but the bladder was distended. A No. 7 catheter passed carefully drew off ten ounces of offensive urine, and during the process a finger in the wound detected a very thin covering to the membranous portion of the urethra, but no rupture.

On December 6th he was free from pain, expression less anxious, urine ammoniacal, and a calculus detected in the bladder.

Dec. 7th. - There is incontinence of urine during coughing. Wound promising to heal well; allowed to use catheter himself.

Jan. 2nd, 1875.-Wound, which, when granulating, presented a natural dissection of the anterior perineal space, is now nearly closed. Urine clearer and less offensive, but still alkaline. To get up.

7th.-The ground still feels numb to the feet, and there is also a want of sensation about the buttocks ; feet always cold; urine free from albumen, alkaline, muco-pus and crystals of triple phosphates present.

Feb. 1st.- Has been up daily since last report; can get about fairly well with a stick, but walks on the heels. At 3.30 P.M. lithotomy was performed laterally; the stone, a small friable one, with a soft nucleus, broke in extraction, and the remaining portions were removed with a scoop, and the bladder syringed out through the wound.

7th.-Bowels, confined since the operation, have to-day responded to an aperient without pain. Ordered dilute nitric and phosphoric acids, with tincture of muriate of iron; allowed solid food; has passed water through the penis on coughing.

12th.-A pint of water passed by natural channel ; urine contains ropy mucus, with pus-cells and plenty of triple phosphate crystals. Ordered dilute nitric acid, tincture of henbane, and decoction of pareira brava.

16 th. - Water all by the right way; is anxious to get up.

20th.-Is up and well; passes his water naturally with a fair stream, requiring to bear down a little. Urine is acid on emission; it dribbles at night sometimes. To pass water frequently, in order to keep the bladder as empty as possible.

March 4th.-Passes water in good stream ; urine is clear, acid, and free from all deposit; his paralysed condition is unaltered, and the tendency to constipation is well met by half-drop doses of croton oil in a pill. 